\title{
Research of a MFC-based wireless communication system
}

\author{
Yanlei $\mathrm{Ma}^{1, \mathrm{a}}$, Lina $\mathrm{Hao}^{2, \mathrm{~b} *}$, Zhen $\mathrm{An}^{3, \mathrm{c}}$, Tong $\mathrm{Zhu}^{4, \mathrm{~d}}$ \\ ${ }^{1,2,3,4}$ School of Mechanical Engineering and Automation, Northeastern University, Shenyang, China \\ 110819 \\ a 441014277@qq.com \\ b haolina@me.neu.edu.cn (corresponding author), \\ c981221164@qq.com
}

Keywords: MFC framework; remote video transmission; wireless serial communication; the AVICap class

Abstract. To realize point to point communication between two devices with a long distance, this paper designs a wireless video transmitting module working at $1.2 \mathrm{GHz}$ and a data transmitting module working at $433 \mathrm{MHz}$. We also write a control program using MFC (Microsoft Fundamental Classed). At last, the system is verified via tests for a tracked robot which carries a camera and many sensors. The result indicates that this system can communicate with the remote host computer timely.

\section{Introduction}

In real-time environmental monitoring or data acquisition system, the traditional method is to collect the signal from each acquisition node through cable. This makes cabling complex, workload for maintenance large, and work field also greatly restricts cabling. The use of wireless data transmission can save cabling time and largely reduce cost for maintenance. So tasks such as acquisition of environmental parameters under harsh working conditions can be much easier [1].

Currently the most extensive short-distance wireless communications technology are infrared, Bluetooth, WI-Fi, ultra-wideband technology, ZigBee and spread spectrum communication technology. Among them, the infrared, ultra wideband technology and Bluetooth's transmitting distance is only 3 10 meters which can not satisfy remote control's requirement [2]. ZigBee is often used in a multipoint wireless communication system. Although it can be used for point to point wireless communication, it cost too much and maintenance is more difficult compared to spread spectrum communication technology [3]. Spread spectrum communication technology has stable transmission and long linear working distance. The latter factor is indispensable. The representative products of spread spectrum communication technology are $1.2 \mathrm{GHz}$ wireless video transmission devices and wireless serial transmission devices which are chosen to realize the communication between host computer and a distant tracked robot. In this paper, we use MFC to develop a piece of control software. MFC is a programming tool provided by Microsoft [4].

\section{Design of a kind of software on host computer}

The software we design has two modular. One is a wireless video transmitting module; the other is a wireless serial communicating module. These two modules works parallel. They don't disturb each other. Figure 1 shows the structure of the software. 


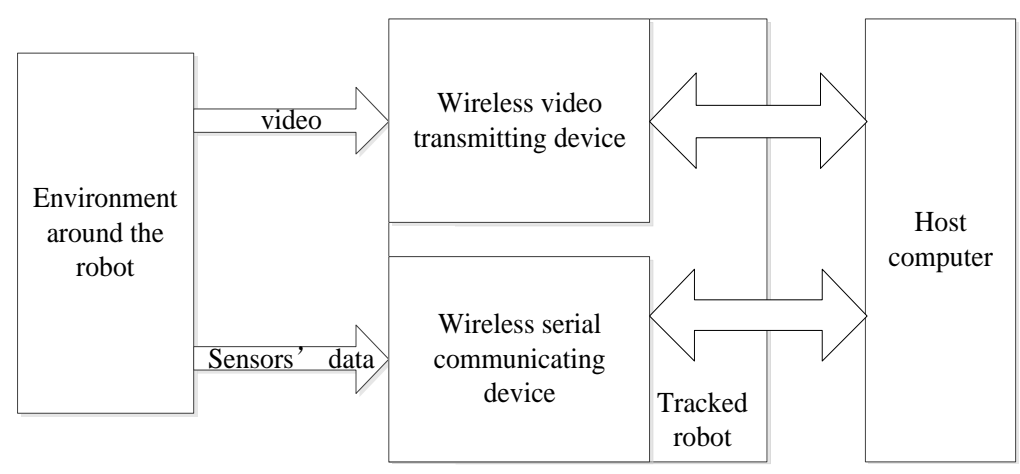

Figure 1 the software's structure

\section{Module of wireless video transmitting.}

Video capture is a process which uses camera to capture an analog signal, transforms it to a digital signal, and then stores the signal or handles the signal in real time. In order to integrate video acquisition and processing [5], this paper uses AVICap class in Microsoft's VFW (video for windows) to capture video in real time and store single image. Specific process is shown in Figure 2

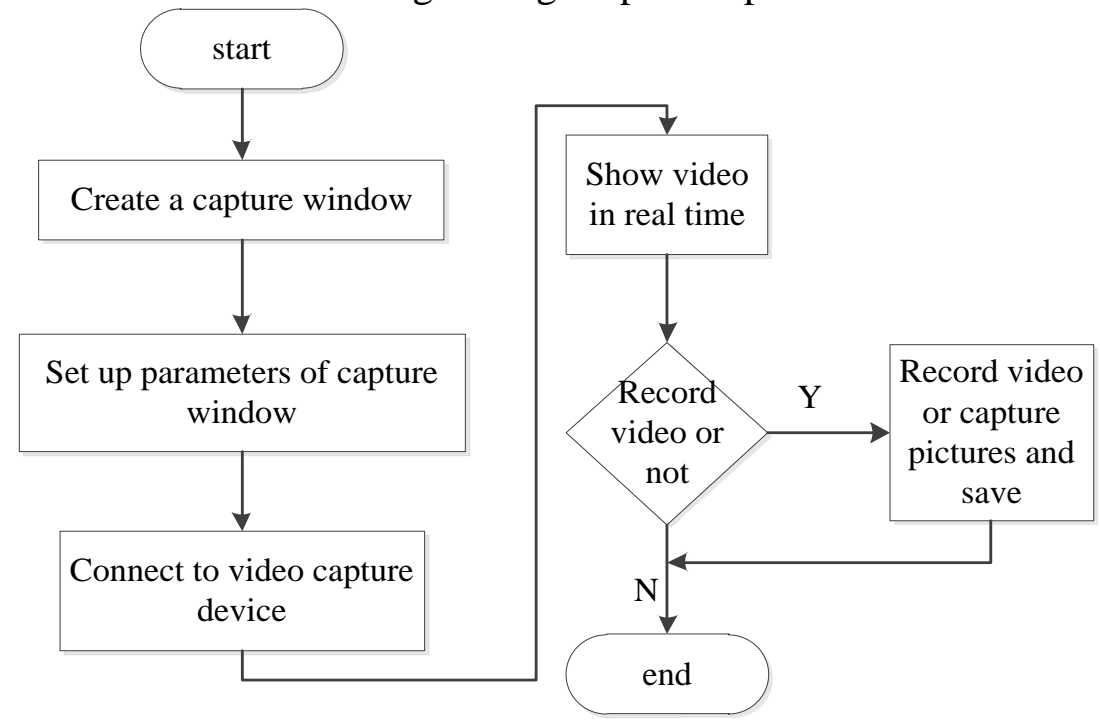

Figure 2 Process for video capture

The specific method of video capture is as follows:

(1) Create a capture window, connect it to the camera

Create a dialog project, use the window class HWND defines a public window handle m_hVideo, create a window with function capCreateCaptureWindow() and connect the window and m_hVideo by capDriverConnect(). capCreateCapture() has eight parameters, representing window's name , window's mode, the $\mathrm{x}$, y coordinates of the window's top left corner, the window's width, height, parent window's handle, the window 's ID number. After the window is created, call the function capSetCallbackOnFrame() to get a structure body of the window which contains detail parameters. So we can make detailed settings for the window's properties. Part of the code is as follows: $m \_h V i d e o=:: c a p C r e a t e C a p t u r e W i n d o w\left(" v i d e o ", W S \_C H I L D \mid W S \_V I S I B L E, 0,0,600,400, m \_h W n d, 0\right)$ capSetCallbackOnFrame(m_hVideo,FrameCallbackProc); capGetDriverDescription(0,szDeviceName,sizeof(szDeviceName),szDeviceVersion,sizeof(szDevice Version));

capDriverConnect(m_hVideo,0); //connect to the camera capPreviewRate(m_hVideo,25);//frame rate 25

capPreview(m_hVideo,TRUE);//preview model

(2) Video capture settings

AVICap has a structural body called CAPTUREPARMS. The structure comprises video capture's setup parameters, such as whether to limit the capture time, capture the video in the foreground or the background, whether single click stops Capture, etc. Declare a CAPTUREPARMS structure named 
CapParam, get the original video capture parameters via function capCaptureGetSetup() [6] and assign to our new structure CapParam, then change CapParam parameters by manually assigning. At last give window's setting parameters, CapParam, back to AVICap using capCaptureSetSetup().

(3) Button's settings on the interface of the software

Select the "button" control from the control Panel, and then drag the control into the dialog then double-click the control to establish the message function of the button and write code in the message function, for example:

capDriverDisconnect(m_hVideo);//disconnect to the camera

(4) Recording and saving video

The AVICap class has relevant functions to help us on the video recording and saving [7]. CapFile-SetCaptureFile() can creates a video file for saving video while capCaptureSequence() is used to send a command to start the video recording. The file's name is the moment that the capture starts. MFC provides a CTime class that contains a member function GetCurrentTime(), which can be used to obtain the current date and time in the format "YY: YY: YY". There is a problem that the file's name should not contain the symbol ":". The time's format need to be changed to "YYXYYXYY".

The code should be written in the message function of "Start recording" button. The way of setting the "stop recording" button is the same, but the message function should be filled with capCaptureStop ( $m \_h$ Video).

(5) preservation of pictures

CapFileSaveDIB() can save a image as a DIB file. CapFileSaveDIB() takes two parameters. The first one is to grab the handle of the window, and the second is the file's path and name. The method of naming is the same to video files.

\section{Module of wireless serial communication.}

Wireless serial communication device needs to connect computer by the serial line to USB converter. Figure 3 shows the process how the host computer communicates with the tracked robot.

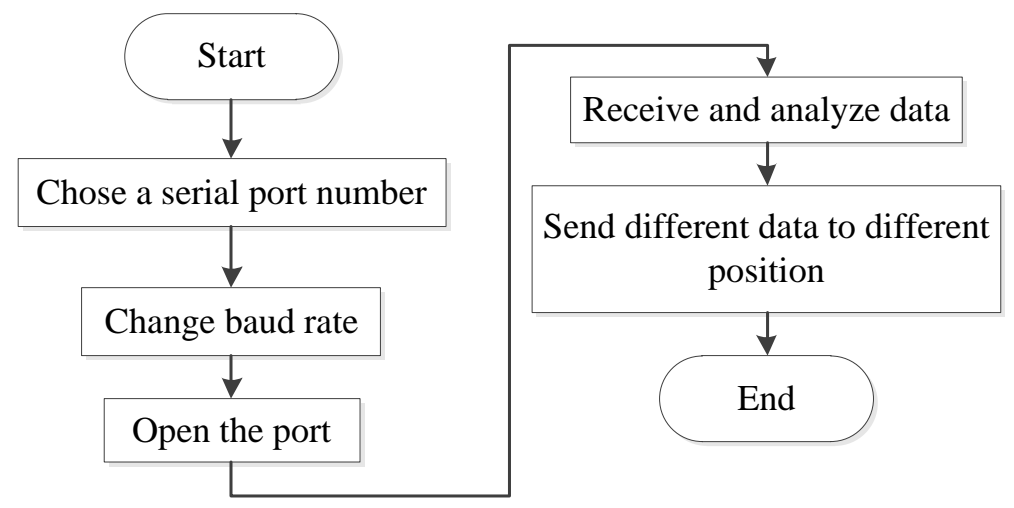

Figure 3 process for wireless serial communication

MSComm is a serial communication control in MFC. A new object, derived from the CMSComm class, needs to be created so that the operation to the serial port can be realized [8]. There is a control called combo box. It has a member function AddString(). By this function various items, such serial port number, can be add to the drop-down menu in the combo box. Radio button is used to represent a series of mutually exclusive options. Only one option can be selected. This paper provides two serial transmission modes, namely "4800, N, 8, 1" and "9600, N, 8, 1". And the message function OnRadio() should be created.

The host computer will receive data continuously once the serial port is open. MSComm provides OnOnCommMscomm() to monitor the receiving buffer. As long as the buffer receiving data, OnOnCommMscomm() will be triggered. However, the data can't be missed, but the wireless transmission device will randomly divide the data. And there is a great delay between two data block. If we use OnOnCommMscomm() to receive the data, we often can not receive a integral package of 
data. So creating a timer to receive data every 1 second is necessary. So serial port's parameters should be set as follow:

m_MSCSet.SetInputMode(1);//read and write data in binary format

m_MSCSet.SetRThreshold(1); //do not trigger OnComm event

m_MSCSet.SetInputLen(0);//read all the data in the buffer

Mscomm provides GetInPut() to read the data in the serial transmitting buffer, but the data is read as format VARIANT. We need to convert it to format CString to analyze and pass the data to the corresponding edit frame by SetDlgItemText(). The data's format is "SA, data 1, data 2 ... data n,”

The code for analyzing and passing data is as follow:

for $(k=0 ; k<l e n ; k++) / /$ this circulation is used to parse and pass data

\{

if $\left(\right.$ rxdata $\left.[k]=={ }^{\prime} S^{\prime}\right)\{k=k+2 ;\}$

if(rxdata[k]!=',')

\{

$\operatorname{pos} 0+=r x d a t a[k]$;

\}

else

\{

SetDlgItemText(i,pos0);

$\operatorname{pos} 0=" "$;

i++;

\}

\}

\section{Experiment}

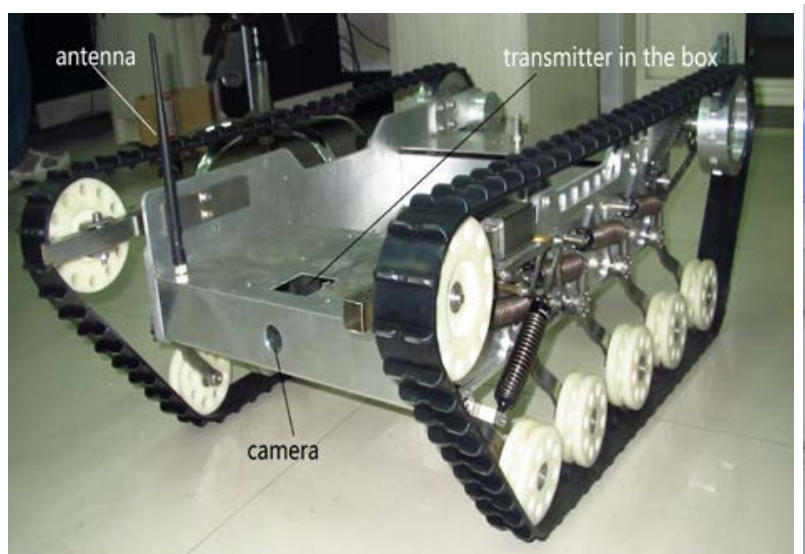

Figure 4 the tracked robot carrying video transmitter

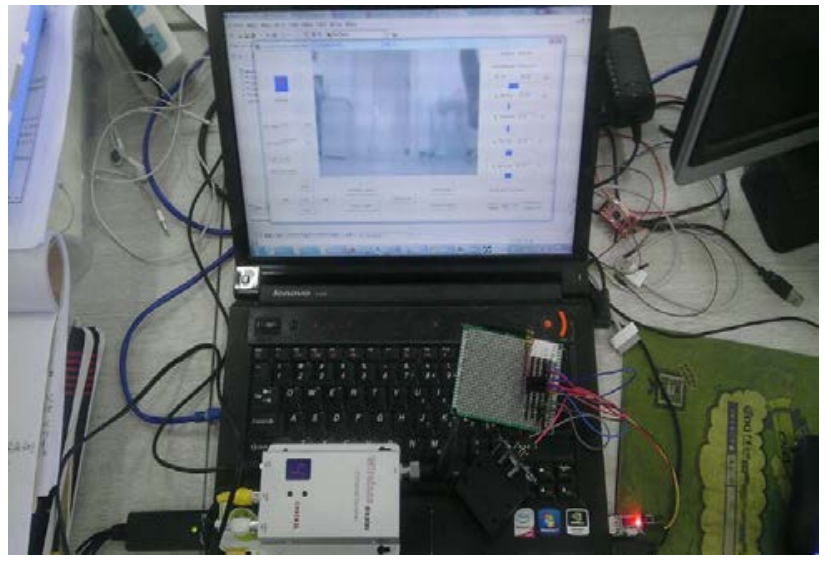

Figure 5 two receivers and the host computer
then let a track robot, which is loaded with the video and serial transmitter, walk on the fifth floor. The result proves that wireless serial port's transmitting distance can be 1000 meters, but if there is a concrete wall in the path, the electromagnetic wave will decay rapidly [9]. And the transmission distance reduces to 100 meters .Although the wireless video transmission device have higher working frequency and it's penetrating ability is stronger, it is difficult to bypass large obstacles[10]. If there is a concrete wall in the path, the transmitting distance drops to 100 meters. However, in an effective distance, the system can transmits clear and accurate information back to the computer. Figure 5 shows the software's interface on the host computer.

\section{Conclusion and discussion}

To collect information around the tracked robot and send them to the remote host computer, this paper compares the current wireless communication technology, establishes a wireless data 
transmitting strategy, and programmes a kind of control software. The studies and experiments above prove that this system can collect visual information in front of the tracked robot and the environmental data around the robot then transmit it to the host computer 100 meters away. The video transmission module works in real-time while the wireless serial communication module sends data every second. This system satisfies the requirement that the timely communication between the robot and the remote host computer.

\section{Acknowledgement}

This work was sponsored by Science and Technology Plan Project of Shenyang(No. F13-181-9-00), Science and Technology Plan Project of Shenyang(No.F13-316-1-74), the Chinese Defense Advance Research Program of Science and Technology, China under Grant (No. 62501040412), LiaoningBaiQianWan Talents Program(No.2013921069), Talent Resources Development Special F unds of Shenyang(2010010403), The National High Technology Research and Development Program of China (No.2015AA042302).

\section{References}

[1] Chaofeng Lian. Rescue robot's wireless communication system under coal mine [D].Xi'an: Xi'an university of science and tecnology, 2010:1-2

[2] Fei Ding. Wireless technology's application based on ZigBee [J]. Jiangsu communication technology, 2006, 22(5): 24-27

[3] Anzar Mahmood. A review of wireless communication for smart grid [J]. Renewable and Sustainable Energy Reviews, 2015, (41):248-260

[4] Zhongzhi Che. Analysis for MFC’s framework [J]. Agriculture Information. 2010. (9):145-147

[5] Yanyan Ye. Video acquisition and processing system based on VC++. [D] Wuhan: Wuhan University of Technology, 2013.6:3-4

[6] Mengting zhou. Remote video capture based on VFW [J].Technology Research. 2014, (5):26-30

[7] Jianghua Li. real-time video capture based on VFW [J]. Science and Technology application, 2005, 32(9) :53-55

[8] Feng Wei. MSComm serial port communication in embedded systems[J]. Lianyungang Technical College Institute. 2014,27(4):12-15

[9] lan F.Akyildiz. Signal propagation techniques for wireless underground communication networks [J]. Physical Communication, 2009, 2(3):167-183

[10] Shijuan Yang. Underground wireless communication network system [D]. Shandong University of Science and Technology, 2006:1 